\title{
Sharp Tractability Borderlines for Finding Connected Motifs in Vertex-Colored Graphs
}

\author{
Michael R. Fellows ${ }^{1}$, Guillaume Fertin ${ }^{2}$, Danny Hermelin ${ }^{\star 3}$, and Stéphane Vialette ${ }^{4}$ \\ 1 School of Electrical Engineering and Computer Science, \\ The University of Newcastle, Calaghan NSW 2308 - Australia \\ mike.fellows@cs.newcastle.edu.au \\ 2 Laboratoire d'Informatique de Nantes-Atlantique (LINA), FRE CNRS 2729 \\ Université de Nantes, 2 rue de la Houssinière, 44322 Nantes Cedex 3 - France \\ fertin@lina.univ-nantes.fr \\ 3 Department of Computer Science, University of Haifa, \\ Mount Carmel, Haifa 31905 - Israel \\ danny@cri.haifa.ac.il \\ 4 Laboratoire de Recherche en Informatique (LRI), UMR CNRS 8623 \\ Faculté des Sciences d'Orsay - Université Paris-Sud, 91405 Orsay - France \\ vialette@lri.fr
}

\begin{abstract}
We study the problem of finding occurrences of motifs in vertex-colored graphs, where a motif is a multiset of colors, and an occurrence of a motif is a subset of connected vertices with a bijection between its colors and the colors of the motif. This problem has applications in metabolic network analysis, an important area in bioinformatics. We give two positive results and three negative results that together draw sharp borderlines between tractable and intractable instances of the problem.
\end{abstract}

\section{Introduction}

Vertex-colored graph problems have numerous applications in bioinformatics: Sandwich problems have applications in DNA physical mapping $[9,16,18]$ and in perfect phylogeny $[11,21]$, while vertexrecoloring problems arise in protein-protein interaction networks and phylogenetic analysis $[10,12$, $22]$. In this paper, we consider another natural vertex-colored graph problem with an interesting application in bioinformatics:

Graph MotiF:

Input: A vertex-colored graph $G$ and a multiset of colors $M$.

Question: Does $G$ have a connected subset of vertices with a bijection between its colors and $M$ ?

The Graph Motif problem was introduced in a slightly more general form by Lacroix, Fernandes, and Sagot (who allowed multiple colors per vertex) in the context of metabolic network analysis, an important area in bioinformatics [20]. There, vertices correspond to chemical compounds or reactions, and edges correspond to interactions between these compounds and reactions. The vertex coloring is used to specify different chemical types or functionality. In this scenario, connected motifs correspond to interaction-related sub-modules of the network which consist of a specific set of chemical compounds and reactions. It is argued in [20] that a method for a rational decomposition of a metabolic network into relatively independent functional subsets is essential for a better understanding of the modularity and organization principles in the network. We refer the reader to $[14,20]$ for more biological background of the problem. We also refer to [19] for related relevant work.

\footnotetext{
* Partially supported by the Caesarea Rothschild Institute.
} 
Graph Motif can also be viewed as a generic pattern-matching problem in graphs. Indeed, if $G$ is a path, then the problem reduces to finding unordered occurrences of a given pattern in a given text. Unordered pattern-matching is recently receiving a lot of attention in the pattern-matching community [3-5], and as far as we know, this is the only paper which considers this scenario in trees, not to mention graphs.

In [20], Graph Motif is proved to be NP-complete even if the given vertex-colored graph is a tree, but fixed-parameter tractable in this case when parameterized by the size of the given motif (i.e. $|M|)$. However, as observed by [20], their fixed-parameter does not apply when the vertexcolored graph is a general graph. For this case they only provided a heuristic algorithm which works well in practice. This motivates us to further investigate the tractability landscape of GRAPH MoTIF, and in particular, to investigate it under different parameters which govern the structure of its input. We give an extensive analysis for GRAPH MoTIF, applying techniques from both classical and parameterized complexity, that unravels sharp borderlines between tractable and intractable instances of the problem. More specifically, we give two algorithms and three hardness results that together imply:

1. For motifs of unbounded size, GraPh MotiF is NP-complete already for trees of maximum degree 3 , even if the motif is a set of colors rather than a multiset. For motifs of logarithmic size (in the number of vertices of $G$ ), the problem is polynomial-time solvable in any general graph.

2. Graph Motif is NP-complete for motifs with 2 colors, even if $G$ is bipartite with maximum degree 4. However, it is polynomial-time solvable in constant treewidth graphs for motifs consisting of any constant number of colors (and arbitrary size). When the number of colors in the motif is taken as a parameter, GRAPH MotIF is W[1]-hard even in case $G$ is a tree.

The rest of the paper is organized as follows. In the reminder of this section we discuss notations that will be used throughout the paper. In Section 2, we give two NP-hardness results that will motive the rest of our discussion. Following this, in Section 3 we present a fixed-parameter algorithm (parameterized by $|M|$ ) that that applies for any general graph. In Section 4 we discuss the case when $G$ has bounded treewidth. Finally, in Section 5, we show that Graph Motif is W[1]-hard on trees when parameterized by the number of colors in $M$.

Basic notation and terminology: Throughout the paper, we use $G=(V(G), E(G))$ to denote our given vertex-colored graph, and $n=|V(G)|$ to denote its order. For a vertex $v \in V(G)$, we use $\chi(v)$ to denote the color of $v$, and for a vertex subset $V \subseteq V(G)$, we let $\chi(V)$ denote the multiset of colors $\bigcup_{v \in V} \chi(v)$. For any vertex subset $V \subseteq V(G)$, we let $G[V]$ denote the subgraph of $G$ induced by $V$, i.e. the subgraph on $V$ along with all edges of $G$ that connect vertices in $V$. We assume w.l.o.g. that $G$ is connected.

A motif $M$ is a multiset of colors. If $M$ is in fact a set rather than a multiset, we say that $M$ is colorful. Given a subset of vertices $V \subseteq V(G),|V|=|M|$, we say that $V$ is colored by the colors of $M$, if $\chi(V)=M$. For $V$ to be an occurrence of $M$, we require not only for $V$ to be colored by the colors of $M$, but also for $G[V]$ to be connected. If this is in fact the case, we say that $M$ occurs at $v$ for any vertex $v \in V$. In these terms, the GRAPH Motif problem is the problem of determining whether a given motif $M$ occurs at any vertex of a given vertex-colored graph $G$. We assume w.l.o.g. that $\chi(v) \in M$ for any $v \in V(G)$.

Our analysis is based both on the classical and parameterized complexity frameworks. Readers unfamiliar with these subjects are referred to $[15,17]$. 


\section{Tight NP-Hardness Results}

As mentioned previously in Section 1, GRAPH MotIF is already known to be NP-complete for trees in [20]. Our aim in this section is to tighten this result by showing that GRAPH Motif remains hard for highly restrictive graph classes, even if we restrict ourselves to motifs which are sets rather than multisets, or to motifs which consist of a small number of colors.

We first consider colorful motifs. Recall that a motif $M$ is colorful if it consists of $|M|$ distinct colors. At first sight, it might seem that occurrences of colorful motifs should be easier to find, at least for certain types of graphs. Unfortunately, the following theorem proves that this is apparently not the case.

Theorem 1. Graph Motif is NP-complete, even if $M$ is colorful and $G$ is a tree of maximum degree three.

Proof. Graph Motif is clearly in NP. To prove NP-hardness, we present a reduction from the well known NP-complete problem 3-SAT [17]. Recall that 3-SAT asks to determine whether a given 3 -CNF formula is satisfiable, that is, whether there is a truth assignment to the boolean variables of the formula, such that the value of the formula under this assignment is 1 . The problem remains hard even if each variable appears in at most three clauses and each literal (i.e. variable with or without negation) appears in at most two clauses [17]. Hence, we restrict ourselves in our proof to formulas of this type.

Let an instance of 3-SAT be given in the form of a 3 -CNF formula $\Phi=c_{1} \wedge \cdots \wedge c_{m}$ over variables $x_{1}, \ldots, x_{n}$ such that $\left|\left\{c_{j} \mid x_{i} \in c_{j}\right\}\right| \leq 2$ and $\left|\left\{c_{j} \mid \bar{x}_{i} \in c_{j}\right\}\right| \leq 2$ for all $1 \leq i \leq n$. We construct an instance for GRAPH MoTIF as follows. The colored graph $G$ initially consists of a path of $n$ vertices, each colored by a distinct color in $1, \ldots, n$. To a vertex colored $i$ in this path, $1 \leq i \leq n$, we connect a new vertex colored $i^{\prime}$. To a vertex colored $i^{\prime}, 1 \leq i \leq n$, we connect a pair of new non-adjacent vertices, both colored $x_{i}$. Conceptually, each vertex in this pair corresponds to a different truth assignment for $x_{i}$. If a truth assignment to variable $x_{i}$ satisfies clause $c_{j}$, we connect a new vertex colored $c_{j}$ to the vertex colored $x_{i}$ which corresponds to this assignment. This is done for every $x_{i} \in\left\{x_{1}, \ldots, x_{n}\right\}$ and every $c_{j} \in\left\{c_{1}, \ldots, c_{m}\right\}$. We conclude our construction by specifying $M$ to be the set of colors $\left\{1, \ldots, n, 1^{\prime}, \ldots, n^{\prime}, x_{1}, \ldots, x_{n}, c_{1}, \ldots, c_{m}\right\}$. A simple example of this construction is given in Fig. 1. Note that $G$ and $M$ are as required by the theorem.

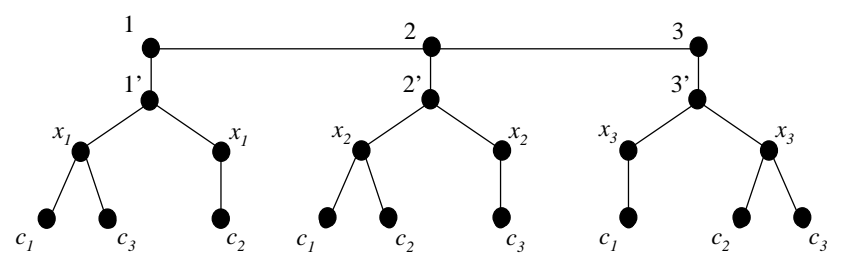

Fig. 1: An example of the construction of $G$ out of a 3-CNF formula which consists of three clauses: $c_{1}=\left(x_{1} \vee x_{2} \vee x_{3}\right)$, $c_{2}=\left(\bar{x}_{1} \vee x_{2} \vee \bar{x}_{3}\right)$, and $c_{3}=\left(x_{1} \vee \bar{x}_{2} \vee \bar{x}_{3}\right)$.

The construction above is clearly polynomial. Hence, to complete the proof, we are left to show that $M$ occurs in $G$ if and only if $\Phi$ is satisfiable. For the first direction, assume that there exists a truth assignment $\phi$ which satisfies $\Phi$. Let $N \subseteq V(G)$ be the subset of vertices in $G$ which are colored by the colors in $\left\{1, \ldots, n, 1^{\prime}, \ldots, n^{\prime}\right\}$, and let $X \subseteq V(G)$ be the subset of vertices which correspond to assignment $\phi$. Hence, $X$ consists of $n$ vertices which are colored by the colors in $\left\{x_{1}, \ldots, x_{n}\right\}$, 
and $N \cup X$ induces a connected subgraph. Since $\phi$ satisfies every clause in $\Phi$, by construction of $G$ there is a vertex colored $c_{j}$ in the neighborhood of $X$ for every $1 \leq j \leq m$. In other words, there exists $C \subseteq N(X)$ which is colored by the colors in $\left\{c_{1}, \ldots, c_{m}\right\}$. It follows that $V=N \cup X \cup C$ is connected and is colored by the colors of $M$, and therefore is an occurrence of $M$ in $G$.

For the converse direction, assume there exists an occurrence $V$ of $M$ in $G$. Let $X \subseteq V$ be the vertices colored by the colors in $\left\{x_{1}, \ldots, x_{n}\right\}, C \subseteq V$ be the vertices colored by the colors in $\left\{c_{1}, \ldots, c_{m}\right\}$, and $\phi$ the truth assignment corresponding to $X$. By construction, a vertex colored $c_{j}$ is connected in $G$ to a vertex colored $x_{i}$ if and only if the truth assignment corresponding to this vertex satisfies clause $c_{j}$. Since $C$ contains all colors in $\left\{c_{1}, \ldots, c_{m}\right\}$, and since vertices in $C$ are connected only to vertices in $X$, it follows that $\phi$ satisfies every clause in $\Phi$, and so it satisfies $\Phi$ itself.

Theorem 1 implies that for motifs of unbounded cardinality, there are not many interesting special cases of Graph Motif left that become polynomial-time solvable. Note that if $G$ is a tree of maximum degree two, then $G$ is actually a path, and GraPH Motif becomes trivial (simply search through all subpaths of length $k$ ). Other cases where $G$ is restricted to special subclasses of trees (e.g. caterpillars) become easily polynomial-time solvable as well. However, the motif in the construction above is not only of unbounded size, it also consists of an unbounded number of colors. One might hope that for motifs which consist of only a small number of colors, GRAPH MотIF would become polynomial-time solvable. The following theorem shows that this is not the case in a very sharp sense.

Theorem 2. Graph Motif is NP-complete, even if $M$ consists of two colors, and $G$ is bipartite with maximum degree four.

Proof. We reduce from the ExACT Cover By 3-Sets (X3C) problem, which is known to be NPcomplete [17]. Recall that, given a set $X=\left\{x_{1}, x_{2}, \ldots, x_{3 q}\right\}$ and a collection $S=\left\{s_{1}, s_{2}, \ldots, s_{n}\right\}$ of 3 -element subsets of $X$, the X3C problem asks to determine whether there exists an exact cover of $X$ in $S$, i.e. a sub-collection $C \subseteq S$ such that every element of $X$ is included in exactly one subset $s_{i} \in C$. The problem is hard even if each element of $X$ appears in at most three sets of $S$ [17], so we restrict ourselves in the proof to instances of this type.

Let $\langle X, S\rangle$ be an arbitrary instance of the X3C problem with $\left|\left\{s_{j} \in S \mid x_{i} \in s_{j}\right\}\right| \leq 3$ for all $x_{i} \in X$. We show how to construct a motif $M$ and a colored graph $G$ in such a way that there exists an exact cover of $X$ in $S$ if and only if $M$ occurs in $G$. First, we define $M$ so as it contains $2 n+3 q$ white elements and $q$ black elements. Then, we define $G$ by $V(G)=X \cup S \cup S^{\prime} \cup S^{\prime \prime}$ and $E(G)=E_{1} \cup E_{2} \cup E_{3} \cup E_{4}$, where $S^{\prime}=\left\{s_{1}^{\prime}, s_{2}^{\prime}, \ldots, s_{n}^{\prime}\right\}$ and $S^{\prime \prime}=\left\{s_{1}^{\prime \prime}, s_{2}^{\prime \prime}, \ldots, s_{n}^{\prime \prime}\right\}$ are dummy copies of $S$, and $E_{1}, E_{2}, E_{3}, E_{4}$ are defined by: $E_{1}=\left\{\left\{x_{i}, s_{j}\right\} \mid x_{i} \in s_{j}\right\}, E_{2}=\left\{\left\{s_{i}, s_{i}^{\prime}\right\} \mid 1 \leq i \leq n\right\}$, $E_{3}=\left\{\left\{s_{i}^{\prime}, s_{i}^{\prime \prime}\right\} \mid 1 \leq i \leq n\right\}$, and $E_{4}=\left\{\left\{s_{i}^{\prime \prime}, s_{i+1}^{\prime}\right\} \mid 1 \leq i \leq n-1\right\}$. The vertices of $X \cup S^{\prime} \cup S^{\prime \prime}$ are colored white and the vertices of $S$ are colored black. It is easily seen that $G$ and $M$ are as required by the theorem, and that our construction can be carried out in polynomial time.

Let us now argue that there exists an exact cover $C \subseteq S$ of $X$ if and only if $M$ occurs in $G$. For the first direction, suppose that there exists an exact cover $C \subseteq S$ of $X$. Consider the subset of vertices $V=X \cup C \cup S^{\prime} \cup S^{\prime \prime}$. First note that $V$ consists of $q=|C|$ black vertices and $2 n+3 q=\left|X \cup S^{\prime} \cup S^{\prime \prime}\right|$ white vertices. Second, since $C$ is a cover of $X$, every vertex of $X$ is connected to some vertex in $C$, and $C$ is connected to $S^{\prime} \cup S^{\prime \prime}$, so $V$ itself is connected. It follows that $V$ is an occurrence of $M$, and $M$ occurs in $G$.

Conversely, suppose that there exists an occurrence $V \subseteq V(G)$ of $M$ in $G$. Observe that $M$ contains $2 n+3 q$ white elements, and since exactly $2 n+3 q$ vertices of $G$ are colored white, we 


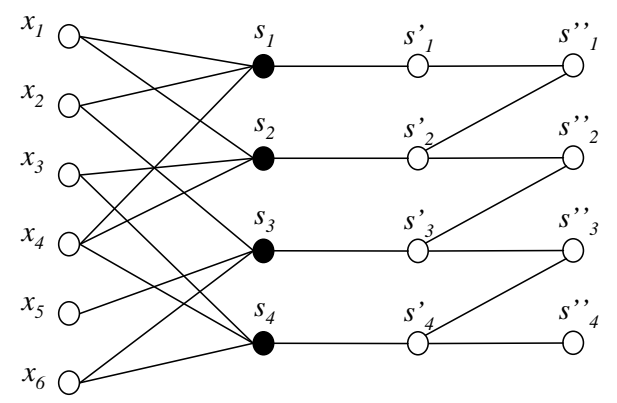

Fig. 2: The construction of $G$ out of an instance for X3C: $X=\left\{x_{1}, \ldots, x_{6}\right\}$ and $S=\left\{s_{1}, \ldots, s_{4}\right\}$. The 3 -sets are $s_{1}=\left\{x_{1}, x_{2}, x_{4}\right\}, s_{2}=\left\{x_{1}, x_{3}, x_{4}\right\}, s_{3}=\left\{x_{2}, x_{5}, x_{6}\right\}$, and $s_{4}=\left\{x_{3}, x_{4}, x_{6}\right\}$.

must have $X \cup S^{\prime} \cup S^{\prime \prime} \subset V$. The remaining $q$ vertices in $V$ are $q$ black vertices from $S$. By construction, we do not have an edge between two vertices of $X$, nor between a vertex of $X$ and a vertex of $S^{\prime} \cup S^{\prime \prime}$. Therefore, since $V$ is connected, each vertex of $X$ has to be adjacent to at least one vertex in $V \cap S$. But $|X|=3 q$ and each vertex in $S$ is connected to exactly 3 vertices in $X$. Then it follows that no two vertices of $V \cap S$ share a common neighbor in $X$, and $C=V \cap S$ is an exact cover of $X$ in $S$.

\section{A General Fixed-Parameter Algorithm}

We now turn to show that GRAPH MotIF is fixed-parameter tractable for parameter $k=|M|$ on any general graph. More specifically, we present an $\mathcal{O}\left(2^{\mathcal{O}(k)} n^{2} \lg n\right)$ algorithm for the problem, which implies that Graph Motif for motifs of $\mathcal{O}(\lg n)$ size is polynomial-time solvable. This is in striking contrast to the sharp hardness results given in the previous section. Our algorithm is based on the color-coding technique introduced by Alon et al. [2], whose derandomized version crucially relies on the notion of perfect hash families:

Definition 1 (Perfect Hash Family). A family $\mathcal{F}$ of functions from $V(G)$ to $\{1, \ldots, k\}$ is perfect if for any subset $V \subseteq V(G)$ of $k$ vertices there is a function $f \in \mathcal{F}$ which is one-to-one on $V$.

Suppose $M$ has an occurrence $V$ in $G$, and suppose we are provided with a perfect family $\mathcal{F}$ of functions from $V(G)$ to $\{1, \ldots, k\}$. Since $\mathcal{F}$ is perfect, we are guaranteed that at least one function in $\mathcal{F}$ assigns $V$ with $k$ distinct labels. Let $f \in \mathcal{F}$ be such a function. For a given $L \subseteq\{1, \ldots, k\}$, we define $\mathcal{M}_{L}(v)$ to be the family of all motifs $M^{\prime} \subseteq M,\left|M^{\prime}\right|=|L|$, for which there exists an occurrence $V^{\prime}$ with $v \in V^{\prime}$, such that the set of (unique) labels that $f$ assigns to $V^{\prime}$ is exactly $L$. Since $M$ occurs in $G$, we know that $M \in \mathcal{M}_{\{1, \ldots, k\}}(v)$ for some $v \in V(G)$. Hence, to determine whether $M$ occurs in $G$, we apply dynamic programming to compute $\mathcal{M}_{L}(v)$ for all $v \in V(G)$ and $L \subseteq\{1 \ldots, k\}$.

Fix $L$ to be some subset of $\{1, \ldots, k\}$, and let $v$ be any vertex of $G$. Our goal is to compute $\mathcal{M}_{L}(v)$ assuming $\mathcal{M}_{L^{\prime}}(u)$ has been previously computed for every vertex $u \in V(G)$ and any $L^{\prime} \subseteq L \backslash\{f(v)\}$. The straightforward approach is to consider small motifs occurring at neighbors of $v$. However, a motif occurring at $v$ might be the union of motifs occurring at any number of neighbors of $v$, and so this approach might require exponential running time in $n$. We therefore present an alternative method for computing $\mathcal{M}_{L}(v)$, which we call the batch procedure, that uses an even more naive approach, but one that requires exponential-time only with respect to $k$. Notice that while the motifs computed by the batch procedure are in general multisets of colors, the batch procedure always considers sets of distinct labels. 


\section{Batch Procedure $(L, v)$ :}

- Define $\mathcal{M}$ to be the family of all pairs $\left(M^{\prime}, L^{\prime}\right)$ such that $M^{\prime} \subseteq M \backslash\{\chi(v)\}, L^{\prime} \subseteq L \backslash\{f(v)\}$, and $M^{\prime} \in \mathcal{M}_{L^{\prime}}(u)$ for some $u \in N(v)$.

- Run through all pairs of $\left(M^{\prime}, L^{\prime}\right),\left(M^{\prime \prime}, L^{\prime \prime}\right) \in \mathcal{M}$ and determine whether $M^{\prime} \cup M^{\prime \prime} \subseteq M \backslash\{\chi(v)\}$, and whether $L^{\prime} \cap L^{\prime \prime}=\emptyset$. If there is such a pair, add $\left(M^{\prime} \cup M^{\prime \prime}, L^{\prime} \cup L^{\prime \prime}\right)$ to $\mathcal{M}$ and repeat this step. Otherwise, continue to the next step.

- Set $\mathcal{M}_{L}(v)$ to be all motifs $M^{\prime} \cup\{\chi(v)\}$ where $\left(M^{\prime}, L^{\prime}\right) \in \mathcal{M}$ and $L^{\prime}=L \backslash\{f(v)\}$.

Lemma 1. For any $v \in V(G)$ and $L \subseteq\{1, \ldots, k\}$, the batch procedure correctly computes $\mathcal{M}_{L}(v)$ assuming $\mathcal{M}_{L^{\prime}}(u)$ is given for every neighbor $u$ of $v$ and every subset of labels $L^{\prime} \subseteq L \backslash\{f(v)\}$.

Proof. Let $\mathcal{M}$ be the family of pairs computed by the batch procedure. Consider any pair $\left(M^{\prime}, L^{\prime}\right) \in$ $\mathcal{M}$ with $L^{\prime}=L \backslash\{f(v)\}$. By construction, $M^{\prime} \subseteq M \backslash\{\chi(v)\}$ and can be written as $M^{\prime}=M_{1}^{\prime} \cup \cdots \cup M_{\ell}^{\prime}$, where each $M_{i}^{\prime}, 1 \leq i \leq \ell$, is a motif that has an occurrence $V_{i}^{\prime}$ which includes a neighbor of $v$. Furthermore, each $V_{i}^{\prime}$ is labeled by a unique set of labels $L_{i}^{\prime}$ such that $L_{i}^{\prime} \cap L_{j}^{\prime}=\emptyset$ for all $1 \leq j \leq \ell$, $j \neq i$. It follows that all the $V_{i}^{\prime}$ s are pairwise disjoint, and that $\{v\} \cup V_{1}^{\prime} \cup \cdots \cup V_{\ell}^{\prime}$ is connected. Hence, $M^{\prime} \cup\{\chi(v)\}$ has an occurrence in $G$ which is labeled by $L^{\prime} \cup\{f(v)\}=L$, and so $M^{\prime} \cup\{\chi(v)\} \in \mathcal{M}_{L}(v)$.

On the other hand, consider a motif $M^{\prime} \cup\{\chi(v)\} \in \mathcal{M}_{L}(v)$. Then by definition, $M^{\prime} \cup\{\chi(v)\}$ has an occurrence $V^{\prime} \cup\{v\}$ such that the set of labels that $f$ assigns $V^{\prime} \cup\{v\}$ is $L$. Let $V_{1}^{\prime}, \ldots, V_{\ell}^{\prime}$ be the connected components of the induced subgraph $G\left[V^{\prime}\right]$. Since $V^{\prime} \cup\{v\}$ is connected, every $V_{i}^{\prime}$, $1 \leq i \leq \ell$, includes a neighbor of $v$. Furthermore, letting $L_{i}^{\prime}$ denote the set of labels that $f$ assigns $V_{i}^{\prime}$ for every $1 \leq i \leq \ell$, we have $L^{\prime} \subseteq L \backslash\{f(v)\}$ and $L_{i}^{\prime} \cap L_{j}^{\prime}=\emptyset$ for all $1 \leq i, j \leq \ell$. It is now easy to see that the batch procedure will eventually compute the pair $\left(M^{\prime}, L \backslash\{f(v)\}\right)$ in its second step, and hence $M^{\prime} \cup\{\chi(v)\}$ will be added to $\mathcal{M}_{L}(v)$ in its final step.

Lemma 2. Given a labeling function $f: V(G) \rightarrow\{1, \ldots, k\}$, one can use the batch procedure iteratively in order to determine in $\mathcal{O}\left(2^{5 k} \mathrm{kn}^{2}\right)$ time whether there is an occurrence of $M$ which is distinctly labeled by $f$.

Proof. To prove the lemma, let us first analyze the complexity of a single invocation of the batch procedure. In its first step, the batch procedure searches through at most $2^{k} n$ motifs families, each consisting of at most $2^{k}$ motifs. Hence, this step requires $\mathcal{O}\left(2^{2 k} k n\right)$ time. For the second step, notice that number of distinct motif and label-subset pairs is bounded by $2^{2 k}$, and so the number of times the second step is repeated is also bounded by this term. Since each iteration of this step can be computed in $\mathcal{O}\left(2^{2 k} k\right)$ time, it follows that the second step requires $\mathcal{O}\left(2^{4 k} k\right)$ time. Accounting also for the third step, the total time of one invocation of the batch procedure is therefore $\mathcal{O}\left(2^{4 k} k+2^{2 k} k n\right)=\mathcal{O}\left(2^{4 k} k n\right)$.

It now can easily be seen that due to Lemma 1 , one needs to invoke the batch procedure at most $2^{k} n$ times in order to obtain $\mathcal{M}_{L}(v)$ for every vertex $v \in V(G)$ and every label subset $L \subseteq\{1, \ldots, k\}$. It follows that in $\mathcal{O}\left(2^{5 k} k n^{2}\right)$ time one can obtain all necessary information to determine whether $M$ has an occurrence which is distinctly labeled by $f$, and so the lemma follows.

Note that in case $M$ is colorful, the vertex-coloring of $G$ distinctly colors any occurrence of $M$, and therefore, in this case we can determine whether $M$ occurs in $G$ within the time complexity given in Lemma 2. For general multiset motifs, we use the result of Alon et al. [2] who show how to efficiently construct a family $\mathcal{F}$ of $\mathcal{O}\left(2^{\mathcal{O}(k)} \lg n\right)$ functions from $V(G)$ to $\{1, \ldots, k\}$ which is perfect. This construction builds on an earlier slightly less efficient construction of [23] and requires $\mathcal{O}\left(2^{\mathcal{O}(k)} n \lg n\right)$ time. Using this and Lemma 2, we obtain a $\mathcal{O}\left(2^{\mathcal{O}(k)} n^{2} \lg n\right)$ algorithm for GRAPH Motif. 
Theorem 3. Graph Motif can be solved in $\mathcal{O}\left(2^{\mathcal{O}(k)} n^{2} \lg n\right)$ time.

Proof. The algorithm uses a perfect family $\mathcal{F}$ of $\mathcal{O}\left(2^{\mathcal{O}(k)} \lg n\right)$ functions from $V(G)$ to $\{1, \ldots, k\}$. Such a family exists, and can be constructed in $\mathcal{O}\left(2^{\mathcal{O}(k)} n \lg n\right)$ time. Given any $f \in \mathcal{F}$, we can use the batch procedure to to determine whether there is any occurrence of $M$ which is distinctly labeled by $f$ in $\mathcal{O}\left(2^{\mathcal{O}(k)} n^{2}\right)$ time (Lemma 2). Since $\mathcal{F}$ is perfect, any occurrence of $M$ in $G$ is guaranteed to be distinctly labeled by at least one labeling function $f \in \mathcal{F}$, and so by exhaustively searching through all functions in $\mathcal{F}$, our algorithm can determine whether $M$ occurs in $G$ within the time bound promised above.

\section{Bounded Treewidth Graphs}

The treewidth parameter of graphs [24] plays a central role in designing exact algorithms for many NP-hard graph problems $[6-8,13]$. Among numerous frameworks developed over the years, we adopt the parsing mechanism developed for bounded treewidth graphs in [1]. For motifs which consist of a constant number of colors $c$ and graphs with treewidth smaller than some constant $\omega$, we present a polynomial-time algorithm with running time $\mathcal{O}\left(n^{2 c \omega+2}\right)$. This should be compared with the sharp hardness result of Theorem 2. Moreover, our algorithm is also a fixed-parameter algorithm for parameters $\omega$ and $k$ which outperforms the algorithm of the previous section when the treewidth of $G$ is sufficiently small. Due to space limitations, we only present a sketch of our result.

Theorem 4. Let $\omega$ be any positive constant. Then GRAPH Motif can be solved in $\mathcal{O}\left(n^{2 c \omega+2}\right)$ time, when $G$ has treewidth less than $\omega$ and $M$ consists of c colors.

Proof (sketch). The proof is sketched as follows. We employ the parsing operator point of view on bounded treewidth. In particular, we use the notion of $\omega$-boundaried graphs, where an $\omega$-boundaried graph is no more than a graph with $\omega$ distinguished vertices, each distinctly labeled by a label in $\{1, \ldots, \omega\}$, which are referred to as boundary vertices. The boundary vertices, together with $\omega-$ operators, allow the construction of $\omega$-boundaried graphs from smaller $\omega$-boundaried graphs. The $\omega$-operators are:

1. The null operator $\emptyset$ which creates the trivial boundaried graph with isolated vertices.

2. The binary operator $\oplus$ that takes the disjoint union of two $\omega$-boundaried graphs and then identifies the $i$ th boundary vertex of the first graph with the $i$ th boundary vertex of the second graph.

3. The unary operator that introduces a new isolated vertex and makes this the new vertex 1 of the boundary. The old vertex 1 is removed from the boundary but not from the graph.

4. The unary operator that adds an edge between vertex 1 and vertex 2 of the boundary.

5. Unary operators that permute the labels of the boundary vertices.

A parse tree is an at-most binary rooted tree with labels corresponding to $\omega$-operators. The leaves are labeled with $\emptyset$, the internal unary nodes are labeled with unary operators, and the internal binary nodes are labeled with the binary operator $\otimes$. Each rooted subtree of a parse tree corresponds to an $\omega$-boundaried graph, where the graph at each parent is obtained by applying the corresponding operator of the parent on the $\omega$-boundaried graph(s) of its child(ren). We say that a parse tree parses an $\omega$-boundaried graph $H$, if $H$ corresponds to the $\omega$-boundaried graph of the root. We extend this definition to any graph, by simply assuming that the final parsing operator removes all vertices from the boundary. Any graph of treewidth less than $\omega$ can be parsed by a parse tree with $\mathcal{O}(\omega n)$ nodes $[1]$. 
Define a checklist item for a $w$-boundaried graph to consist of the following information: (1) A partition $\pi$ of the set of boundary vertices. Let $X$ denote the set of boundary vertices, and write $\pi=\left(X_{1}, \ldots, X_{r}\right)$ where $r \leq \omega$, and the $X_{i}$ denote the sets of the partition $\pi$. (2) A motif family $\mathcal{M}_{\pi}=\left(M_{0}, M_{1}, \ldots, M_{r}\right)$ of length $r+1$, where each $M_{i}$ is non-empty except maybe $M_{0}$ and $M_{i} \subseteq M$. (Note that the number of distinct checklist items is at most $\omega^{\omega}\left(n^{c}\right)^{\omega}=w^{w} n^{c \omega}$, where a better bound is given by replacing $w^{w}$ with $\operatorname{Bell}(\omega)$, the number of distinct partitions of an $\omega$-element set.)

We say that a checklist item $\alpha$ as above is positive for a $\omega$-boundaried graph $A$ if there is a set of $r+1$ vertex-disjoint subsets $V_{0}, \ldots, V_{r} \subseteq V(A)$ satisfying the following conditions:

1. $V_{0} \cap X=\emptyset$.

2. For $i=1, \ldots, r, V_{i} \cap X=X_{i}$.

3. For $i=0, \ldots, r, V_{i}$ is an occurrence of $M_{i}$ in $A$

Define the inventory $\operatorname{inv}(A)$ of the $w$-boundaried graph $A$ to be the set of all checklist items that are positive for $A$.

Claim 1. Whether a motif occurs in a $\omega$-boundaried graph $A$ can be determined from $i n v(A)$ in time linear in the size of the inventory.

Our algorithm proceeds as follows. It first computes a parse tree of $G$, and then computes, from the leaves up to the root, the inventories of the $\omega$-boundaried graphs corresponding to the nodes of the $\omega$ parse tree. Let $A$ be the trivial boundary graph obtained by the null operator $\emptyset$. In this base case, $\operatorname{inv}(A)$ consists of single checklist item with a partition $\pi=\left(X_{1}, \ldots, X_{r}\right)$ that partitions the boundary vertices into singletons, and motif family $\mathcal{M}_{\pi}=\left(\emptyset, M_{1}, \ldots, M_{r}\right)$ where $M_{i}$ consists of the color of the single boundary vertex in $X_{i} \in \pi$, for all $i=1, \ldots, r$. For boundary graphs obtained by unary operations, computing the inventory is almost equally easy.

Claim 2. One can compute $i n v(o p(A))$ from $i n v(A)$.

We proceed to describe the computation for the $\otimes$ operator. Let $A$ and $B$ be two boundaried graphs over the same boundary vertex set $X$. If $\alpha \in i n v(A)$ and $\beta \in i n v(B)$ then we define the checklist item $\alpha \oplus \beta$ as follows. As per the definition of a checklist item, we must give two pieces of information to describe $\alpha \oplus \beta$ : (1) a partition $\pi_{\alpha \oplus \beta}$ of $X$, and (2) a motif family $\mathcal{M}_{\pi_{\alpha \oplus \beta}}$ for this partition. Let $\pi_{\alpha}\left(\pi_{\beta}\right)$ denote the partition of $X$ for the checklist item $\alpha(\beta)$. Let $\equiv_{\alpha}\left(\equiv_{\beta}\right)$ be the equivalence relation on $X$ defined by $\pi_{\alpha}\left(\pi_{\beta}\right)$. The partition $\pi_{\alpha \oplus \beta}$ corresponds to the reflexive and transitive closure of the relation $\equiv_{\alpha} \cup \equiv_{\beta}$. The motif family $\mathcal{M}_{\pi_{\alpha \oplus \beta}}$ is obtained by adding and subtracting colors of the motifs of $\mathcal{M}_{\pi_{\alpha}}$ and $\mathcal{M}_{\pi_{\beta}}$ in the natural way.

Claim 3. We can compute $i n v(A \oplus B)$ as $\{\alpha, \beta, \alpha \oplus \beta \mid \alpha \in \operatorname{inv}(A), \beta \in \operatorname{inv}(B)\}$.

Hence given a parse tree of $G$, we have to perform at most $\omega n$ such "multiplications of inventories". Since each inventory has size bounded by $\omega^{\omega} n^{c \omega}$, and since a single multiplication between two inventories requires $\mathcal{O}(n)$ time, this gives a running time of $\mathcal{O}\left(n^{2 c \omega+2}\right)$ for all inventory multiplications. Since the computation on the $\otimes$ operator requires more time then the computation on any other operator, the entire algorithm runs within this time bound.

Showing that our algorithm is a fixed-parameter algorithm for parameters $\omega$ and $k$ involves pretty much the same analysis. The only difference is that here we bound the total number of distinct checklist items by $\omega^{\omega}\left(2^{k}\right)^{\omega}=w^{w} 2^{k \omega}$. 


\section{On Trees and Motifs With Bounded Number of Colors}

Although Theorem 4 gives a nice complementary result to the sharp hardness result of Theorem 2, it still leaves a certain gap. In the following section we aim to close this gap, by proving that GRAPH MoTIF, parameterized by the number of colors $c$ in $M$, is W[1]-hard for trees. Essentially, this means that unless unlikely collapses occur in parameterized complexity theory, there is no fixed-parameter algorithm for parameter $c$, even in the restricted case of trees. We refer readers unfamiliar with the concept of parameterized reductions to [15].

Theorem 5. The Graph Motif problem, parameterized by the number of colors $c$ in the motif, is $\mathrm{W}[1]$-hard for trees.

Proof. We present a reduction from CLIQUE which is known to be W[1]-hard [15]. Recall that in Clique we are given a simple graph $H$ and an integer $\kappa$, the parameter, and we are asked to determine whether $H$ has a subset of $\kappa$ vertices which are pairwise adjacent. Given an instance $\langle H, \kappa\rangle$ of Clique, we describe a rooted tree $G=T$ colored with $1+\kappa+2 \kappa(\kappa-1)+\left(\begin{array}{c}\kappa \\ 2\end{array}\right)$ colors. We let $m$ denote the number of edges of $H$, i.e. $m=|E(H)|$.

- The root of $T$ is colored $a$.

- The root has $\kappa \cdot|V(H)|$ children organized into $\kappa$ groups $S(1) \ldots S(\kappa)$. The group of $|V(H)|$ children $S(i)$ consists of the nodes $s(i, u)$, where $u \in V(H)$. The color of each node in $S(i)$ is $b(i)$.

- From each node $s(i, u)$ hang $\kappa-1$ groups of paths. The groups are $P(i, u, j)$ for every $j \in$ $\{1 \ldots, \kappa\} \backslash\{i\}$. There is one path $p(i, u, j, v) \in P(i, u, j)$ for each edge $\{u, v\} \in E(H)$ that is incident to $u$ in $H$.

The path $p(i, u, j, v)$ begins with a vertex colored $c(i, j)$ and ends with a vertex colored $d(i, j)$, and otherwise consists of some number $m(i, u, j, v)$ of internal vertices colored by $e(i, j)=e(j, i)$. There is an important detail to note here. If $i<j$, then $c(i, j)$ and $c(j, i)$ are different colors, whereas $e(i, j)$ and $e(j, i)$ are the same color. We call the colors $e(i, j)$ the edge colors. The number $m(i, u, j, v)$ is calculated as follows. Number the edges in $E(H)$ from 1 to $m$, letting $l(u v)$ denote the number assigned to the edge $\{u, v\} \in E(H)$. We define:

$$
m(i, u, j, v)= \begin{cases}l(u v) & i<j \\ 3 m-l(u v) & i>j .\end{cases}
$$

The motif $M$ consists of one of each of every color other than the edge colors, and $3 m$ elements colored by each edge color. Thus, $M$ consists of $c=1+\kappa+2 \kappa(\kappa-1)+\left(\begin{array}{c}\kappa \\ 2\end{array}\right)$ different colors, and $|M|=1+\kappa+2 \kappa(\kappa-1)+3 m\left(\begin{array}{c}\kappa \\ 2\end{array}\right)$. This completes the construction of our instance $\langle(G, M), c\rangle$ for Graph Motif.

We claim that $H$ has a subset of $\kappa$ pairwise adjacent vertices if and only if $T$ has a subtree $T^{\prime}$ which is an occurrence of $M$. Suppose that the vertices $v_{1}, \ldots, v_{\kappa}$ are pairwise adjacent in $H$. The subtree $T^{\prime}$ consists of:

- The root which is colored $a$.

- The $\kappa$ children of the root $s\left(i, v_{i}\right)$ for all $1 \leq i \leq \kappa$, where $s\left(i, v_{i}\right)$ is colored $b(i)$.

- The $\kappa(\kappa-1)$ paths $p\left(i, v_{i}, j, v_{j}\right)$. The path $p\left(i, v_{i}, j, v_{j}\right)$ begins with a node colored $c(i, j)$ and ends with a node colored $d(i, j)$ for all $1 \leq i, j \leq \kappa, i \neq j$. Note that the path $p\left(i, v_{i}, j, v_{j}\right)$ is pendant from $s\left(i, v_{i}\right)$ since $v_{i}$ and $v_{j}$ are adjacent in $H$. Together, the two complementary paths $p\left(i, v_{i}, j, v_{j}\right)$ and $p\left(j, v_{j}, i, v_{i}\right)$ contain $3 m$ nodes colored $e(i, j)$. 

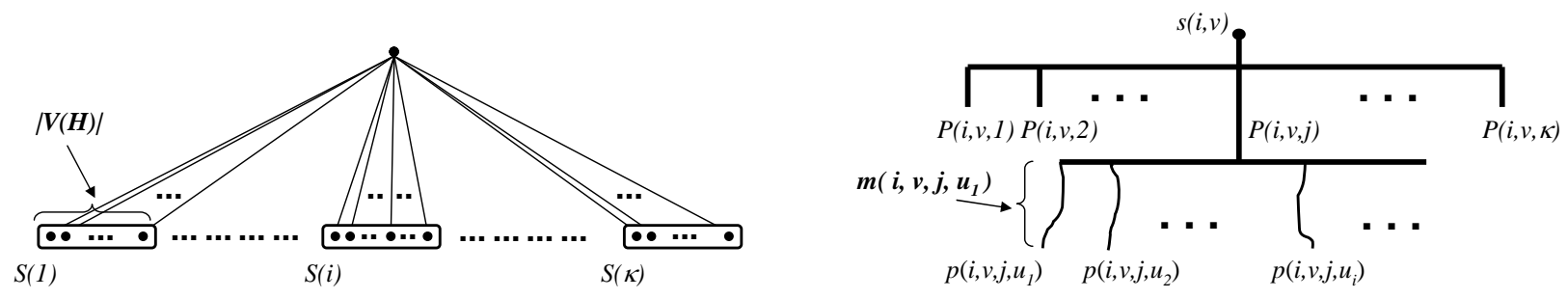

Fig. 3: A schematic description of the tree constructed in the reduction. On the left, the first two levels of the tree are depicted. On the right, the group of paths which hang from the node $s(i, v) \in S(i)$.

In the other direction, suppose that the subtree $T^{\prime}$ of $T$ is an occurrence of $M$. Then $T^{\prime}$ must include the root of $T$, since it is the only node colored $a$. Furthermore, $T^{\prime}$ must contain exactly one node in each of the groups $S(i), 1 \leq i \leq \kappa$, since nodes in each $S(i)$ are all colored $b(i)$. Suppose these nodes are $s\left(1, v_{1}\right), \ldots, s\left(\kappa, v_{\kappa}\right)$. We argue that the vertices $v_{1}, \ldots, v_{\kappa}$ are pairwise adjacent in $H$.

In order for $T^{\prime}$ to be an occurrence of $M$ in $T, T^{\prime}$ must contain exactly one pendant path in each of the groups of paths $P\left(i, v_{i}, j\right)$ for any $1 \leq i, j \leq \kappa, i \neq j$, and nothing further. To see this, note that $T^{\prime}$ must contain at least one path in each of the groups of paths $P\left(i, v_{i}, j\right)$ in order for $T^{\prime}$ to contain a node colored $d(i, j)$. But containing one such path prevents $T^{\prime}$ from including any nodes of other paths in $P\left(i, v_{i}, j\right)$, else $T^{\prime}$ would contain too many nodes of color $c(i, j)$.

It follows that for any pair of indices $i, j$ with $1 \leq i<j \leq \kappa, T^{\prime}$ includes exactly two paths $p\left(i, v_{i}, j, x\right)$ and $p\left(j, v_{j}, i, y\right)$ that contain nodes of color $e(i, j)=e(j, i)$. Since $M$ contains exactly $3 m$ elements colored by $e(i, j)$, it follows that $x=v_{j}$ and $y=v_{i}$, since $p\left(i, v_{i}, j, v_{j}\right)$ and $p\left(j, v_{j}, i, v_{i}\right)$ are the only two paths in $T$ with nodes colored $e(i, j)$ that together have exactly $3 m$ nodes of this color. But then, by the construction of $T, v_{i}$ and $v_{j}$ must be adjacent in $H$.

\section{References}

1. K.R. Abrahamson and M.R. Fellows. Finite automata, bounded treewidth, and well-quasiordering. In Graph Structure Theory (ed. N. Robertson and P. Seymour), pages 539-564, 1993.

2. N. Alon, R. Yuster, and U. Zwick. Color coding. Journal of the ACM, 42(4):844-856, 1995.

3. A. Amir. Asynchronous pattern matching. In Proceedings of the 17th annual symposium on Combinatorial Pattern Matching (CPM), pages 1-10, 2006.

4. A. Amir, A. Apostolico, G.M. Landau, and G. Satta. Efficient text fingerprinting via parikh mapping. Journal of Discrete Algorithms, pages 409-421, 2003.

5. A. Amir, Y. Aumann, G. Benson, A. Levy, O. Lipsky, E. Porat, S. Skiena, and U. Vishne. Pattern matching with address errors: rearrangement distances. In Proceedings of the 17th annual ACM/SIAM Symposium On Discrete Algorithms(SODA), pages 1221-1229, 2006.

6. S. Arnborg. Efficient algorithms for combinatorial problems on graphs with bounded decomposability. A survey. BIT Numerical Mathematics, 25(1):2-23, 1985.

7. S. Arnborg and A. Proskurowski. Linear time algorithms for NP-hard problems restricted to partial $k$-trees. Discrete Applied Mathematics, 23:11-24, 1989.

8. H.L. Bodlaender. A tourist guide through treewidth. Acta Cybernetica, 11:1-23, 1993.

9. H.L. Bodlaender and L.E. de Fluiter. Intervalizing k-colored graphs. In Proceedings of the 22nd International Colloquium on Automata, Languages and Programming (ICALP), pages 87-98, 1995.

10. H.L. Bodlaender, M.R. Fellows, M.A. Langston, M.A. Ragan, F.A. Rosamond, and M. Weyer. Kernelization for convex recoloring. In Proceedings of the 2nd workshop on Algorithms and Complexity in Durham (ACiD), pages 23-35, 2006.

11. H.L. Bodlaender, M.R. Fellows, and T. Warnow. Two strikes against perfect phylogeny. In Proceedings of the 19th International Colloquium on Automata, Languages and Programming (ICALP), pages 273-283, 1992. 
12. B. Chor, M.R. Fellows, M.A. Ragan, F.A. Rosamond, and S. Snir. Connected coloring completion for general graphs: Algorithms and complexity - Manuscript. 2007.

13. D.G. Corneil and J.M. Keil. A dynamic programming approach to the dominating set problem on $k$-trees. SIAM Journal on Algebraic and Discrete Methods, 8(4):535-543, 1987.

14. Y. Deville, D. Gilbert, J. Van Helden, and S.J. Wodak. An overview of data models for the analysis of biochemical pathways. Briefings in Bioinformatics, 4(3):246-259, 2003.

15. R. Downey and M. Fellows. Parameterized Complexity. Springer-Verlag, 1999.

16. M.R. Fellows, M.T. Hallett, and H.T. Wareham. DNA physical mapping: Three ways difficult. In Proceedings of the 1st annual European Symposium on Algorithms (ESA), pages 157-168, 1993.

17. M.R. Garey and D.S. Johnson. Computers and Intractability: A Guide to the Theory of NP-Completeness. Freeman, New York, 1979.

18. M. Golumbic, H. Kaplan, and R. Shamir. On the complexity of DNA physical mapping. Advances in Applied Mathematics, 15:251-261, 1994.

19. T. Ideker, R.M. Karp, J. Scott, and R. Sharan. Efficient algorithms for detecting signaling pathways in protein interaction networks. Journal of Computational Biology, 13(2):133-144, 2006.

20. V. Lacroix, C.G. Fernandes, and M.-F. Sagot. Reaction motifs in metabolic networks. In Proceedings of the 5th international Workshop on Algorithms in BioInformatics (WABI), pages 178-191, 2005.

21. F.R. McMorris, T.J. Warnow, and T. Wimer. Triangulating vertex-colored graphs. SIAM Journal on Discrete Mathematics, 7(2):296-306, 1994.

22. S. Moran and S. Snir. Convex recolorings of strings and trees: Definitions, hardness results and algorithms. In Proceedings of the 9th international Workshop on Algorithms and Data Structures (WADS), pages 218-232, 2005.

23. J. Naor and M. Naor. Small-bias probability spaces: Efficient constructions and applications. In Proceedings of the 25th annual ACM Symposium on Theory Of Computing (STOC), pages 213-223, 1990.

24. N. Robertson and P. D. Seymour. Graph minors. II. Algorithmic aspects of tree-width. SIAM Journal of Algorithms, 7:309-322, 1986. 